\title{
Whatsapp: A Trend Setter in Mobile Communication among Chennai Youth
}

\author{
Ms. Jisha $\mathrm{K}^{1}$, Dr. Jebakumar ${ }^{2}$ \\ ${ }^{I}$ (Department of Journalism \& Mass Communication,Faculty of Science \& Humanities, SRM University, India) \\ ${ }^{2}$ (Department of Visual Communication, Thiru Vi Ka College, Bharathidasan University, India)
}

\begin{abstract}
Today the mobile application, WhatsApp has become a part of youth's life. It has become a communication application that youth find it difficult to be without, whether they agree it or not. They get in touch with friends and family, and use it for fulfilling their information, entertainment and educational purposes. They use it for the exchange of emotional intimate content and also to co-ordinate their everyday life. The groups that youth create with friends and colleagues help them to share their ideas, thoughts and emotions. The youth in India made a gradual shift from all social networking sites to WhatsApp in a short span of time. To investigate the importance of WhatsApp among youth, a study was conducted among 100 college going students in Chennai region, especially who use smart phones, in the age group of between 18 to 23. An online survey was conducted to draw findings. It was found that students spent 8 hours per day on using WhatsApp and remain online almost 16 hours a day. All the respondents agreed that they are using WhatsApp for communicating with their friends. They also exchange images, audio and video files with their friends using WhatsApp. It was also proved that the only application that the youth uses when they are spending time on their smart phone is WhatsApp.
\end{abstract}

Key Words: Smart phones, Mobile Communication, Mobile App, Inter-personal communication, WhatsApp \& Youth.

\section{Introduction} Einstein

"It has become exceedingly obvious that our technology has exceeded our humanity." -Albert

The world of communication is ever changing due to the developments in the field of science and technology. People feel so proud that they are depending upon technology in their day to day life. Right after getting up in the morning till their last work before sleep, they make use of technology. To fulfill their need of communication, the most often used technology driven media are computers and mobile phones. Today's generation is too much obsessed with luxury and their life is literally taken away by the use of new media. The trend of multi-tasking, an activity of instant messaging while doing homework or during class lectures has become a usual phenomenon in youngsters' life today. The trend of using new media 24/7 was a phenomenon seen in the foreign countries, but now this has become a part people in India too. Today, it's rare to see a youngster without a gadget in hand. They are completely involved in using new media in one form or the other. The performance and capability of mobile phones have changed a lot. The use of smart phones among youth in India has increased in a short span of time. The reason for this is the element of interactivity and the varieties of application that it provides for the youth. Some of the smart phone Apps that youth uses today are games, navigation software, e-book readers, services providing news, weather feeds, and other apps that need internet connectivity like facebook, viber, youtube, WhatsApp etc. Out of all these applications, WhatsApp has become more popular among youth. The current study is focused on youth in Chennai region, a metropolis in India.

\begin{abstract}
About Chennai
Chennai, also known as Madras, is the capital of the state, Tamil Nadu, India, which has a rich tradition and civilization with a blend of both old and the new. It is a metropolis, and the fourth largest state in India. Chennai is the biggest industrial and commercial centre in South India and a major cultural, economic and educational centre. It is the sixth most populous city in India.
\end{abstract}

\subsection{Objectives}

This study examines the usage of WhatsApp mobile application among the youth in Chennai region. The objectives for the study are

1. To investigate the intensity of WhatsApp use among youth in Chennai region.

2. To explore the various uses of WhatsApp among its users.

3. To find out the frequency and interactivity of WhatsApp among its users. 
4. To explore the features of WhatsApp used the most by youngsters.

5. To find out whether youth is satisfied with the mobile app, WhatsApp.

\subsection{Research Questions}

1. How frequent does youngsters in Chennai region use WhatsApp messenger?

2. What are the purposes fulfilled by WhatsApp messenger for youngsters in Chennai region?

3. Whether the youngsters of Chennai region are happy using WhatsApp messenger in their day to day life in fulfilling their personal and academic needs?

\section{Review Of Literature}

There are many studies done on WhatsApp and youth both in Indian and international scenario. A study entitled "The impact of WhatsApp messenger usage on students performance in tertiary institutions in Ghana" by Johnson Yeboah and George Dominic Ewur published in the journal of Education \& Practice in the year 2014, has concentrated on the negative aspects of WhatsApp. Their study proved that the usage of WhatsApp has impacted negatively on the performance of students in Ghana. Some of the findings of the study were the following. WhatsApp usage has led to lack of concentration during lectures, consumes much of students study time, distracts students from completing their assignments, destroys students' spellings and grammatical construction of sentences.

Another study entitled "Smartphone application usage amongst students at a South African University",done in the year 2012, assesses the usage of smartphone applications specifically social networking applications amongst the users in South African University. The study proved that students remain online for 16 hours a day and spends an average of 5 hours per day on their smart phones interacting with others through social networking applications.

An article "Indian Youth prefers WhatsApp, Facebook over SMS" published on June 16, 2013, in The Times Of India Online newspaper. The survey was conducted by Tata Consultancy Services in the years 20122013. The study was conducted covering 17,500 high school students across 14 Indian cities. The study revealed that today's youth are collaborating through social networking tools and building virtual communities aided by affordable bandwidth and smart devices. The survey proved that almost $70 \%$ of the students possess smart phones and have started utilizing the full potential of smart phones.

The article titled "WhatsApp could help Facebook retain youth in countries like India" published online on The Indian Express.com, dated February 21, 2014, talks about the acquisition of WhatsApp by Facebook. The reason they state in the article is increasing number of smart phone users in India and the proliferation of cheaper smart phones and data plans in India. Recent reports suggest that the youth have been moving away from Facebook. WhatsApp, on the other hand, is the fastest growing messaging app. The acquisition is a way of future-proofing growth, especially in a young country like India. Youth in India have started spending more time on messaging apps.

\subsection{About The App Whatsapp}

WhatsApp,started in the year 2009, with the tag line "Simple. Personal. Real time messaging," is a mobile messaging app which allows the users to exchange messages without having to pay for SMS. The WhatsApp messenger was purposely created by Brian Acton and Jan Koum (2009) to make communication and the distribution of multimedia messaging more easily and faster. WhatsApp works with internet connectivity and helps its users to stay in touch with friends and relatives in the contact list. Apart from making its users getting connected with each other, it also helps them to create groups, send unlimited images, video and audio messages. Studies have proved that WhatsApp is the most popular instant messenger service used by youth today. Young people who gives more importance to friendship, social lives and family relationships make use of WhatsApp in a large scale. The popularity of WhatsApp among youth has brought a huge profit among service providers since it works on internet data plan. The reason why WhatsApp is so popular among youth is because, it allows them to send unlimited texts to their friends and family members without any cost other than their internet data plan that they already uses in their smart phones. The application is so easy to use after downloading. It shows you who all are using WhatsApp in your contacts and also helps to invite their friends who are yet to download and use. Then they can start messaging, sharing audio files, video files, updating status, etc.

\subsection{Introduction}

\section{Methodology}

The research design enables the researcher to find out appropriate answers to the research questions posed. The purpose of this section is to discuss the method adopted for this study. The aim of this research is to observe and understand WhatsApp usage among Chennai youth. The researcher made use of both primary and 
secondary data, gathering information from various journal articles and internet websites. Primary date was collected using a questionnaire. The questionnaire was well designed with both open ended and close ended questions. An Online survey was conducted in order to find out results.

\subsection{Research methods}

The study uses online survey method and is restricted to youngsters in Chennai region. A questionnaire was developed with both close-ended and open ended questions to assess the demographics of youngsters, their smart phone details, service provider details, ranking of mobile as a necessity of life, ranking of WhatsApp features, and intensity of usage. The open ended questions gave the samples a chance to express their views about WhatsApp messenger and to list out some of the features that they like the most in the app. This gave the researcher to gather more information regarding WhatsApp messenger and youth's use of its features that would not have been said with closed ended questions. The researcher used judgemental sampling to identify the samples for the study. Students in Chennai region, who possess smart phones and are users of WhatsApp messenger were taken for the study.

\subsection{Sample Szie}

The population under study was youth, especially college going students in the state of Chennai, India. Since the population was so high and the researcher was unable to get data from the whole population, questionnaire was distributed to 100 college students in the age group of 18-23. The respondents for the study were students who were users of smart phones and WhatsApp.

\section{Data Collected \& Results}

Demographics including the age, course of study and the year of study were collected. Users were asked to rank the level of usage of WhatsApp messenger on a scale of 1 to 10.

To determine the usage of WhatsApp among youth, they were asked about the number of messages send and received in a day. The average time spent on WhatsApp was also asked for. To determine the intensity of usage of WhatsApp, the number of conversations, the number of people with whom they interacted and the time spent on a regular basis were asked.

To determine the popularity of mobile phone brands, the respondents were asked to specify their brand in the questionnaire.

100 questionnaires were administered with 50 boys and 50 girls those who are college going in Chennai region.

Young people in Chennai use WhatsApp mainly for communication and to update their status on a regular basis. The immediate and ubiquitous use for social co-ordination and updating makes more and more youth download the app and use.Many studies in India have proved that youth make use of social networking tools to communicate with their friends and family. Smart devices and online access make them connected with their friends and relatives. The affordability of smart phones and data plans by the service providers have resulted in a great use of WhatsApp among youth. The service providers are competing with others with their cheap/low cost and attractive data plans. Even the mobile phone industry has underwent great price shift. People can buy smart phone at affordable rates. The urge to get connected with their friends and relatives has made the youth become a 24/7 user of WhatsApp in Chennai. The various features available and the great speed in sending and receiving messages are added advantages of WhatsApp.Mobile internet was not that popular among youth until the launch of WhatsApp application. Now $90 \%$ of youth subscribe for mobile internet as the application works through internet access. Mobile providers ensure faster connection in their internet services and offer attractive and affordable schemes. WhatsApp has brought a boom for the mobile internet concept. After the arrival of WhatsApp, many youngsters who were not a user of mobile internet has shifted to the usage of $2 \mathrm{G}$ and $3 \mathrm{G}$, thereby bringing a huge profit for the mobile service providers through their internet usage. The attractive offers that they provide attract even more youngsters to make them avail 24/7 online. The speed that the internet packages offer retains the set of youngsters to be the regular users. After the entry of WhatsApp as a quick messenger, many of the youngsters started purchasing smart phones/android phones, this resulted in a huge sale for the smart phone industry. Many mobile companies started launching smart phones at affordable rates. This also enhanced the sale of smart phones.

Fig 1 deals with the mobile phone service providers details. According to the study,majority of the youngsters use the service provider Airtel followed by Vodafone, DoCoMo, Reliance and BSNL. 35 out of 100 respondents were using Airtel sim followed by Vodafone (30\%), Docomo(15\%), Reliance (11\%) and BSNL $(9 \%)$. 


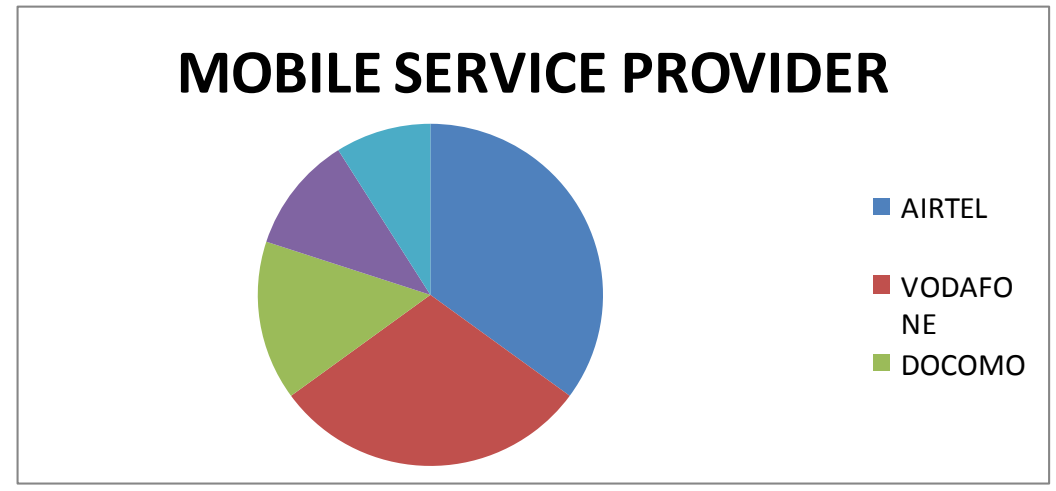

Fig.1. Mobile phone usage details

Fig.2 explains the mobile phone brands used by the youth in Chennai region. The study proved that majority of the youngsters use Samsung mobile phones followed by Nokia, HTC and blackberry. The percentage of respondents using Samsung mobile is $54 \%$ followed by Nokia (19\%), HTC (15\%), and Blackberry (12\%). Samsung is the mobile brand that is popular among the youth in Chennai region.

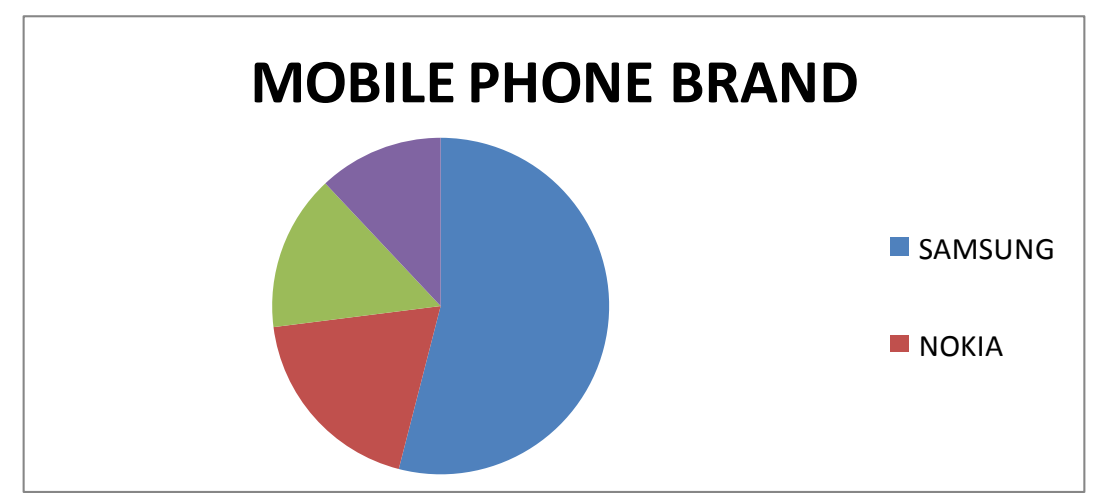

Fig.2. Mobile phone brands used by the people in Chennai region

Majority of the youngsters remain online 24/7 to access WhatsApp, thereby getting in touch with their friends without missing any single message. All the respondents agreed that they use their mobile phone full time to access WhatsApp.

96 out of 100 respondents rated mobile as a necessity of their life in a scale of 1 to 5 . Majority of youngsters use WhatsApp to chat with friends followed by relatives. Majority of the youngsters agreed that they converse with more than 15 people a day through WhatsApp. Majority of respondents rated 5 on a 5 point scale for the features of WhatsApp like instant messaging, group texting and updating profile image and status on a regular basis. The usage of unlimited audio files and video files were given 3 on 5 point scale.

\section{Research Findings}

Youth in Chennai region considers Whatspp as an alternate for text messaging/ social networking sites. They also consider it as a perfect tool for intimate social relationships, as a perfect medium to update their status and images instantly and for exchanging their photos, videos, and audios at a very fast pace, and as a tool for instant communication, with unlimited messages and unrestricted size of sending messages. The respondents also consider it as a means of relaxation and stress relief.

\section{Advantages Of Whatsapp:}

The respondents listed out some of the advantages of WhatsApp.

1. NO COST: You can download the application and use it for one year free of cost to send unlimited messages, audio files, video files, images etc with internet connection. After one year, it demands a payment of $\$ 0.99$ USD per year.

2. EASY TO CHAT: WhatsApp makes chat easy with friends and relatives since you need not invite them to WhatsApp. If they are already in WhatsApp, it automatically gets added to your profile.

3. EASY TO USE : WhatsApp is easy to use since it reduces the users time to log in and log out by giving them access whenever internet is available in just a click in WhatsApp icon. 
4. EASY TO SEND MULTIMEDIA MESSAGES INSTANTLY: WhatsApp enables its users to send images, audio files, video files in a second's time.

5. PRESERVES CHAT HISTORY: Until and unless you delete your chat history, it preserves the messages.

6. ENABLES THE USER TO SEE WHEN THEIR FRIENDS AND RELATIVES LOGGED IN LAST TIME: WhatsApp enables you the view your friend's last log in.

7. QUICK IN STATUS UPDATES AND PHOTO UPLOADS: WhatsApp is very quick when it comes to status updates and photo updates.

8. GROUP CHAT FACILITY: WhatsApp enables youth to chat with their friends and relatives in a group. Youngsters agree that they cant be without WhatsApp too long because they don't want to miss some important conversations made between group friends and relatives.

9. NO GEOGRAPHIC CONSTRAINTS: You can communicate with your friends and relatives no matter where they are located at.

10. GREAT SPEED: the speed in chat messages, photo updation, video and audio updations attract and retains more and more of its users.

The Other Side Of Whatsapp:

1. IMAGE DOWLOADS: Images can be downloaded from the profile pics and used for any purpose.

2. CONSUME LOTS OF SPACE IN MOBILE PHONE:

3. ACCESS TO IMAGES:

4. ACCESS TO ANONYMOUS CONTACTS: Through WhatsApp, people get access to phone numbers and images of anonymous people, just because they are a part of group texting.

5. VISIBLY HECTIC TEXT MESSAGE CULTURE: WhatsApp has encouraged youth to send hectic messages and thereby enhancing the culture of visibly hectic text messages. There is no restriction in the size of text messages that can be send through WhatsApp.

6. LACK OF CONCENTRATION IN CLASS ROOMS: Young people very seldom turn off their WhatsApp, even at colleges, They will be receiving messages every now and then. Due to this they lack concentration in class rooms, and hence wont listen to classes much.

7. LACK OF COMMUNICATION WITH FAMILY MEMBERS AT HOME: The 24/7 online nature in WhatsApp results in lack of communication with the family in the physical world. The youngsters today, are so obsessed with WhatsApp that they rarely communicates with their family.

8. "PART OF ME MISSING" SYNDROME: Without WhatsApp, todays youth feels like something is missing in them if they are not found to be online with friends and relatives. So they keeps their mobile next to their pillow just to check their WhatsApp messages.

Eventhough WhatsApp is having some negative sides, still youth prefers to use it to get connected with their close ones.

\section{Limitations \& Scope For The Study}

- The sample for the study was limited to 100 respondents in Chennai region. The study can be extended to more cities with more respondents.

- The study is restricted to smart phone users. Further researchers can do a research on the popularity of WhatsApp among youth by comparing both smartphone users and non smartphone users.

- The current study has concentrated much on the positive aspects of WhatsApp. In future, researchers can do a study in both pros and cons of WhatsApp.

- The study should have included other apps like Viber and hyke messenger which is gaining popularity in today's scenario.

- There is limited literature review available in Indian context, with reference to WhatsApp usage. Many studies were done on foreign context than in India.

\section{Conclusion}

Many studies conducted in India by taking the youngsters as respondents have revealed that smart devices and unprecedented levels of online access are making the present generation the most connected. Previously, only friends and lovers tend to have strong relationships with intimate conversations. But now whoever you chat through WhatsApp, you develop intimate conversations. WhatsApp has created a sense of belongingness, nearness and intimacy with friends and relatives. It has created a psychological experience of being close and caring. WhatsApp has become so cosy/comfortabe an application among youngsters. Now WhatsApp is being used by youth for creating, sharing and exchanging information. WhatsApp has become one of the reasons for smart phone penetration in India. WhatsApp with its tagline "simple. Personal. Real time 
messaging," has proved that its tagline is very much apt for the brand. No wonder, the brain behind Facebook, Mark Zuckerburg took the ownership of WhatsApp very recently. The main reason is its wide use and popularity among youth itself.

\section{Journal papers:}

\section{References}

[1] Johnson Yeboah, George Dominic Ewur, The impact of Whatsapp messenger on students performance in Tertiary institutions in Ghana, Journal of Education and practice, Vol.5, No.6, 2014.

\section{Theses:}

[2] Kenton O'Hara, Michael Massimi, Richard Harper, Simon Rubens, Jessica Morris, Everyday dwelling with WhatsApp, Microsoft Research Cambridge \& New Experience.

\section{Proceedings Papers:}

[3] Walter UYS, Aadilah Mia, Gary Jeffrey Jansen, Haythem Van Der Schyff ,Michael Andre Josias, Michelle Khusu, Muzaffer Gierdien, Natacha Andrea Leukes, Sulungeka Faltein, Tejas Gihwala,, Tracey-Lee Theunissen, Yaseen Samsodien, Smartphone application usage amongst students at a South African University, IST-Africa 2012 Conference Proceedings Paul Cunningham and Miriam Cunningham (Eds) IIMC International Information Management Corporation, 2012. ISBN: 978-1-905824-34-2.

[4] Karen Church and Rodrigo De Oliveira, Whats up with Whatsapp?;Comparing mobile instant messaging behaviors with traditional SMS, Mobile HCI, page 352-361. ACM, (2013).

\section{Internet References:}

[5] http:/timesofindia.indiatimes.com/tech/social/Indian-youth-prefers-WhatsApp-Facebook-over-SMS-callsSurvey/articleshow/20614937.cms

[6] http://www.newindianexpress.com/cities/chennai/Laptops-are-passe-Chennai-youth-prefer-tabs/2013/06/19/article1641305.ece

[7] http://www.chennai-madras.com/generalinformation.htm 